\title{
Derivation of functional equations for Feynman integrals from algebraic relations
}

\author{
O.V. Tarasov \\ Joint Institute for Nuclear Research, \\ 141980 Dubna, Russian Federation \\ II. Institut für Theoretische Physik, Universität Hamburg, \\ Luruper Chaussee 149, 22761 Hamburg, Germany \\ E-mail: otarasov@jinr.ru
}

ABSTRACT: New methods for obtaining functional equations for Feynman integrals are presented. The application of these methods to finding functional equations for various one- and two-loop integrals is described in detail. It is shown that with the aid of the functional equations Feynman integrals in general kinematics can be expressed in terms of simpler integrals.

Keywords: Renormalization Regularization and Renormalons, Scattering Amplitudes

ArXiv EPRINT: 1512.09024 


\section{Contents}

1 Introduction 1

2 Deriving functional equations from recurrence relations $\quad 2$

3 Algebraic relations between products of propagators 4

3.1 Explicit relations for products of two, three and four propagators 4

$\begin{array}{lll}3.2 & \text { Algebraic relationship for products of any number of propagators } & 7\end{array}$

$\begin{array}{lll}3.3 & \text { Prototypes of functional equations } & 8\end{array}$

4 Some examples of functional equations $\quad 9$

4.1 Functional equation for the one-loop propagator type integral 9

4.2 Functional equations for the one-loop vertex type integral 9

$\begin{array}{lll}4.3 & \text { Functional equations for one-loop box type integrals } & 11\end{array}$

$\begin{array}{lll}4.4 & \text { Functional equation for two-loop vertex type integral } & 12\end{array}$

5 Deriving functional equation by deforming propagators $\quad 14$

$\begin{array}{lll}5.1 & \text { Algebraic relations for products of deformed propagators } & 15\end{array}$

5.2 Functional equation for two-loop vacuum type integral with arbitrary masses 15

6 Conclusions 20

\section{Introduction}

Recently it has been discovered that Feynman integrals obey functional equations $[1,2]$. In these papers, a systematic method for deriving the functional equations was proposed. Different examples of the functional equations were presented in refs. [1-3]. In these articles, only one-loop integrals were considered.

A particular relationship between $N$-point one-loop integrals with nonzero masses connecting integrals with different sets of kinematical arguments was derived in ref. [4]. A detailed consideration of this relationship for the one-loop propagator, vertex- and box-type integrals was presented in [5].

In the present paper, we propose essentially new systematic methods for deriving functional equations. These methods are based on algebraic relations between propagators and they are suitable for deriving functional equations for multiloop integrals. Also, these methods can be used to derive functional equations for integrals with some propagators raised to non-integer powers.

Our paper is organized as follows. Section 2 gives a short review of the method proposed in ref. [1]. 
In section 3, a method for finding algebraic relations between products of propagators is formulated. We describe in detail a derivation of explicit relations for products of two, three and four propagators. Also, the algebraic relation for products of an arbitrary number of proparators is given. These relations are used in section 4 . to obtain functional equations for one- as well as two-loop integrals. In particular, the functional equation for the massless one-loop vertex type integral is presented. Also, the functional equation for the two-loop vertex type integral with arbitrary masses is given.

Section 5 proposes another method for obtaining functional equations. The method is based on finding algebraic relations for 'deformed propagators' and a further conversion of the integrals with 'deformed propagators' to usual Feynman integrals by imposing conditions on the deformation parameters. To perform such conversion, the $\alpha$-parametric representation for both types of integrals is exploited. The method was used to derive a functional equation for the two-loop vacuum type integral with arbitrary masses. From this functional equation we obtained a new hypergeometric representation for the one-loop massless vertex integral.

In conclusion, we formulate our vision of the future applications and developments of the proposed methods.

\section{Deriving functional equations from recurrence relations}

The method for deriving functional equations proposed in ref. [1] is based on the use of a different kind recurrence relations. In particular, in refs. [1-3] the generalized recurrence relations [6] were utilized to obtain functional equations for one-loop Feynman integrals. In general, such recurrence relations connect a combination of some number of integrals $I_{1, n}, \ldots, I_{k, n}$ corresponding to diagrams, say, with $n$ lines and integrals corresponding to diagrams with fewer lines. Diagrams with fewer lines can be obtained by contracting some lines in integrals with $n$ lines. The integrals corresponding to such diagrams depend on fewer kinematical variables and masses compared to integrals with $n$ lines. Such recurrence relations can be written in the following form:

$$
\sum_{j} Q_{j}\left(\left\{m_{i}\right\},\left\{s_{q}\right\}, \nu_{l}, d\right) I_{j, n}=\sum_{k, r<n} R_{k, r}\left(\left\{m_{i}\right\},\left\{s_{m}\right\}, \nu_{l}, d\right) I_{k, r},
$$

where $Q_{j}$ and $R_{k}$ are polynomials depending on masses $m_{i}$, scalar products $s_{r}$ of external momenta, powers of propagators $\nu_{l}$ and parameter of the space time dimension $d$. In the left-hand side of eq. (2.1) we combined integrals with $n$ lines and in the right-hand side integrals with fewer lines.

In accordance with the method of ref. [1], to obtain functional equation from eq. (2.1), one should eliminate terms on the left-hand side by defining some kinematical variables from the set of equations:

$$
Q_{j}\left(\left\{m_{i}\right\},\left\{s_{q}\right\}, \nu_{l}, d\right)=0 .
$$

If there are nontrivial solutions of this system and for these solutions some $R_{k, r}\left(\left\{m_{i}\right\},\left\{s_{m}\right\}, \nu_{l}, d\right)$ are different from zero, then the right-hand side of eq. (2.1) will represent the functional equation. 


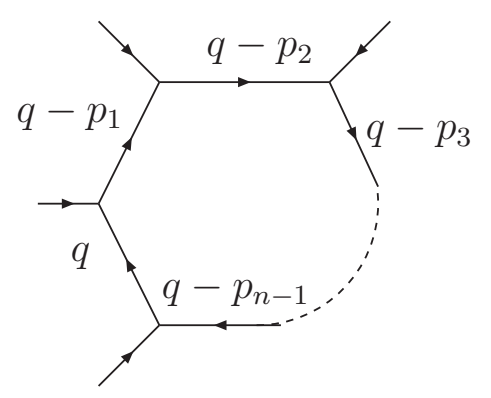

Figure 1. One-loop diagram with $n$ external legs.

As an illustrative example, we consider one-loop integrals with $n$ propagators

$$
I_{n}^{(d)}\left(\left\{m_{j}^{2}\right\},\left\{s_{k l}\right\}\right)=\int \frac{d^{d} q}{i \pi^{d / 2}} \prod_{j=1}^{n} \frac{1}{P_{j}^{\nu_{j}}},
$$

where

$$
P_{j}=\left(q-p_{j}\right)^{2}-m_{j}^{2}+i \eta .
$$

Here as usual an imaginary term $i \eta$ with infinitesimal $\eta>0$ implies a prescription on how to treat the poles of the propagators. The diagram corresponding to this integral is given in figure 1. Different types of recurrence relations for $I_{n}^{(d)}\left(\left\{m_{j}^{2}\right\},\left\{s_{k l}\right\}\right)$ were given in refs. [6, 7].

In particular, in refs. $[6,7]$ the following relation was derived:

$$
\begin{aligned}
& \left(d-\sum_{i=1}^{n} \nu_{i}+1\right) G_{n-1} I_{n}^{(d+2)}\left(\left\{m_{j}^{2}\right\},\left\{s_{k l}\right\}\right)-2 \Delta_{n} I_{n}^{(d)}\left(\left\{m_{j}^{2}\right\},\left\{s_{k l}\right\}\right) \\
& =\sum_{k=1}^{n}\left(\partial_{k} \Delta_{n}\right) \mathbf{k}^{-} I_{n}^{(d)}\left(\left\{m_{j}^{2}\right\},\left\{s_{k l}\right\}\right),
\end{aligned}
$$

where the operators $\mathbf{k}^{-}$shift the index of propagators by one unit $\nu_{k} \rightarrow \nu_{k}-1$,

$$
\begin{aligned}
G_{n-1} & =-2^{n}\left|\begin{array}{cccc}
\left(p_{1}-p_{n}\right)\left(p_{1}-p_{n}\right) & \left(p_{1}-p_{n}\right)\left(p_{2}-p_{n}\right) & \ldots & \left(p_{1}-p_{n}\right)\left(p_{n-1}-p_{n}\right) \\
\left(p_{1}-p_{n}\right)\left(p_{2}-p_{n}\right) & \left(p_{2}-p_{n}\right)\left(p_{2}-p_{n}\right) & \ldots & \left(p_{2}-p_{n}\right)\left(p_{n-1}-p_{n}\right) \\
\vdots & \vdots & \ddots & \vdots \\
\left(p_{1}-p_{n}\right)\left(p_{n-1}-p_{n}\right) & \left(p_{2}-p_{n}\right)\left(p_{n-1}-p_{n}\right) & \ldots & \left(p_{n-1}-p_{n}\right)\left(p_{n-1}-p_{n}\right)
\end{array}\right|, \\
\Delta_{n} & =\left|\begin{array}{cccc}
Y_{11} & Y_{12} & \ldots & Y_{1 n} \\
Y_{12} & Y_{22} & \ldots & Y_{2 n} \\
\vdots & \vdots & \ddots & \vdots \\
Y_{1 n} & Y_{2 n} & \ldots & Y_{n n}
\end{array}\right|, \\
Y_{i j} & =m_{i}^{2}+m_{j}^{2}-s_{i j},
\end{aligned}
$$

Here $p_{i}, p_{j}$ are external momenta going through lines $i, j$, respectively, and $m_{j}$ is the mass attributed to the $j$-th line. The Gram determinant $G_{n-1}$ and modified Cayley determinant $\Delta_{n}$ are polynomials depending on scalar products of external momenta and masses. In the case where all $\nu_{j}=1$ the recurrence relation $(2.5)$ for the first time was derived in refs. [8, 9]. 
It is assumed that these scalar products are made of $d$ dimensional vectors and $G_{n-1}$ and $\Delta_{n}$ are not subject to any restriction or condition specific to some integer values of $d$. Equation (2.5) is written in the form corresponding to eq. (2.1). To eliminate integrals with $n$ lines in the left-hand side of eq. (2.5), the following conditions are to be held:

$$
G_{n-1}=0, \quad \Delta_{n}=0 .
$$

Equation (2.5) is valid for arbitrary kinematical variables and masses. The solution of eqs. (2.8) can be easily done with respect to two kinematical variables or masses. Starting from $n=3$, substitution of such solutions into eq. (2.5) gives nontrivial functional equations [1].

The method for obtaining the functional equations by eliminating complicated integrals from the recurrence relations is quite general. However, for multiloop integrals depending on several kinematical variables, derivation of equations like eq. (2.5) is computationally challenging. In the next sections, we will describe easier and more powerful methods that can be used for deriving the functional equations for multiloop integrals.

\section{$3 \quad$ Algebraic relations between products of propagators}

Setting $\nu_{j}=1$ in eq. (2.5) and imposing conditions (2.8) lead to the following equation:

$$
\sum_{k=1}^{n}\left(\partial_{k} \Delta_{n}\right) \mathbf{k}^{-} I_{n}^{(d)}=0
$$

In eq. (3.1) the integrands of $\mathbf{k}^{-} I_{n}^{(d)}$ are products of $n-1$ propagators depending on different external momenta, i.e. each term in this relation corresponds to the same function but with different arguments. In fact, the functional equations considered in refs. [1-3] are of the same form as eq. (3.1). The question naturally arises: does this relationship hold for integrals or it can be obtained as a consequence of a relationship between integrands?

By inspecting eq. (3.1), one can suggest the following form of the algebraic relation between the products of propagators of integrands:

$$
\prod_{r=1}^{n} \frac{1}{P_{r}}=\frac{1}{P_{n+1}} \sum_{r=1}^{n} x_{r} \prod_{\substack{j=1 \\ j \neq r}}^{n} \frac{1}{P_{j}},
$$

where

$$
P_{j}=\left(k_{1}-p_{j}\right)^{2}-m_{j}^{2}+i \eta .
$$

In what follows we will omit the in term assuming that all masses have such a correction.

Now let us consider in detail a derivation of the algebraic relations for products of 2,3 and 4 propagators.

\subsection{Explicit relations for products of two, three and four propagators}

At $n=2$ relation (3.2) reads:

$$
\frac{1}{P_{1} P_{2}}=\frac{x_{1}}{P_{2} P_{3}}+\frac{x_{2}}{P_{1} P_{3}}
$$


where

$$
P_{1}=\left(k_{1}-p_{1}\right)^{2}-m_{1}^{2}, \quad P_{2}=\left(k_{1}-p_{2}\right)^{2}-m_{2}^{2}, \quad P_{3}=\left(k_{1}-p_{3}\right)^{2}-m_{3}^{2} .
$$

Multiplying both sides of eq. (3.4) by $P_{1} P_{2} P_{3}$ one gets:

$$
P_{3}=x_{1} P_{1}+x_{2} P_{2} .
$$

In what follows it will be assumed that $k_{1}$ is an integration momentum and three vectors $p_{1}, p_{2}, p_{3}$ do not depend on it. Differentiating eq. (3.6) with respect to $k_{1 \mu}$ yields:

$$
k_{1 \mu}-p_{3 \mu}=x_{1}\left(k_{1 \mu}-p_{1 \mu}\right)+x_{2}\left(k_{1 \mu}-p_{2 \mu}\right) .
$$

This equation is equivalent to a system of two equations:

$$
\begin{aligned}
& 1=x_{1}+x_{2}, \\
& p_{3}=x_{1} p_{1}+x_{2} p_{2} .
\end{aligned}
$$

The last equation in (3.8) means that the Gram determinant for the set of three vectors $p_{1}, p_{2}, p_{3}$ is equal to zero. Taking into account eqs. (3.8) another equation follows from eq. (3.6):

$$
x_{1} x_{2} s_{12}-m_{1}^{2} x_{1}-m_{2}^{2} x_{2}+m_{3}^{2}=0 .
$$

Equation (3.9) and the first equation in (3.8) define the system of equations for $x_{1}, x_{2}$ with the solution

$$
x_{1}=\lambda_{2}, \quad x_{2}=1-\lambda_{2},
$$

where $\lambda_{2}$ is the root of the equation

$$
A_{2} \lambda_{2}^{2}+B_{2} \lambda_{2}+C_{2}=0
$$

with

$$
A_{2}=s_{12}, \quad B_{2}=m_{1}^{2}-m_{2}^{2}-s_{12}, \quad C_{2}=m_{2}^{2}-m_{3}^{2} .
$$

This solution can be rewritten in an explicit form:

$$
\begin{aligned}
& x_{1}=\frac{m_{2}^{2}-m_{1}^{2}+s_{12}}{2 s_{12}} \pm \frac{\sqrt{\Lambda_{2}+4 s_{12} m_{3}^{2}}}{2 s_{12}}, \\
& x_{2}=\frac{m_{1}^{2}-m_{2}^{2}+s_{12}}{2 s_{12}} \mp \frac{\sqrt{\Lambda_{2}+4 s_{12} m_{3}^{2}}}{2 s_{12}},
\end{aligned}
$$

where

$$
\Lambda_{2}=s_{12}^{2}+m_{1}^{4}+m_{2}^{4}-2 s_{12} m_{1}^{2}-2 s_{12} m_{2}^{2}-2 m_{1}^{2} m_{2}^{2} .
$$

Now let us find an algebraic relation for the products of three propagators. At $n=3$ eq. (3.2) reads:

$$
\frac{1}{P_{1} P_{2} P_{3}}=\frac{x_{1}}{P_{4} P_{2} P_{3}}+\frac{x_{2}}{P_{1} P_{4} P_{3}}+\frac{x_{3}}{P_{1} P_{2} P_{4}},
$$


where $P_{1}, P_{2}, P_{3}$ are defined in eq. (3.5) and

$$
P_{4}=\left(k_{1}-p_{4}\right)^{2}-m_{4}^{2} .
$$

In complete analogy with the previous case we multiply eq. (3.15) by the product of $P_{1} P_{2} P_{3} P_{4}$ and obtain

$$
P_{4}=x_{1} P_{1}+x_{2} P_{2}+x_{3} P_{3} .
$$

Assuming that $p_{j}, x_{r}$ do not depend on $k_{1}$, and differentiating eq. (3.17) with respect to $k_{1 \mu}$ one gets the equation linear in $k_{1}$. This equation is equivalent to the system of two equations:

$$
\begin{aligned}
& x_{1}+x_{2}+x_{3}=1, \\
& p_{4}=x_{1} p_{1}+x_{2} p_{2}+x_{3} p_{3} .
\end{aligned}
$$

Taking into account eqs. (3.18), (3.19), we obtain from eq. (3.17):

$$
x_{2} x_{3} s_{23}+x_{1} x_{3} s_{13}+x_{1} x_{2} s_{12}-m_{1}^{2} x_{1}-m_{2}^{2} x_{2}-m_{3}^{2} x_{3}+m_{4}^{2}=0 .
$$

Solving eqs. (3.18), (3.20) for $x_{1}, x_{2}$ yields

$$
x_{1}=1-\lambda_{3}-x_{3}, \quad x_{2}=\lambda_{3},
$$

where $\lambda_{3}$ is a solution of the equation

$$
A_{3} \lambda_{3}^{2}+B_{3} \lambda_{3}+C_{3}=0 .
$$

Here

$$
\begin{aligned}
& A_{3}=s_{12}, \\
& B_{3}=x_{3}\left(s_{13}+s_{12}-s_{23}\right)-m_{1}^{2}+m_{2}^{2}-s_{12}, \\
& C_{3}=x_{43}^{2} s_{13}+\left(m_{3}^{2}-m_{1}^{2}-s_{13}\right) x_{3}+m_{1}^{2}-m_{4}^{2} .
\end{aligned}
$$

Let us now turn to the derivation of an algebraic relation for the product of four propagators. At $n=4$ eq. (3.2) reads:

$$
\frac{1}{P_{1} P_{2} P_{3} P_{4}}=\frac{x_{1}}{P_{5} P_{2} P_{3} P_{4}}+\frac{x_{2}}{P_{1} P_{5} P_{3} P_{4}}+\frac{x_{3}}{P_{1} P_{2} P_{5} P_{4}}+\frac{x_{4}}{P_{1} P_{2} P_{3} P_{5}},
$$

where $P_{1}, P_{2}, P_{3}, P_{4}$ are defined in eqs. (3.5), (3.16),

$$
P_{5}=\left(k_{1}-p_{5}\right)^{2}-m_{5}^{2} .
$$

Multiplying eq. (3.24) by the product $P_{1} P_{2} P_{3} P_{4} P_{5}$ we obtain:

$$
P_{5}=x_{1} P_{1}+x_{2} P_{2}+x_{3} P_{3}+x_{4} P_{4} .
$$

As it was in two previous cases, we assume that $p_{j}, x_{r}$ do not depend on $k_{1}$. Differentiating eq. (3.26) with respect to $k_{1 \mu}$, one gets an equation linear in $k_{1}$ from which two equations follow

$$
\begin{aligned}
& x_{1}+x_{2}+x_{3}+x_{4}=1, \\
& p_{5}=x_{1} p_{1}+x_{2} p_{2}+x_{3} p_{3}+x_{4} p_{4} .
\end{aligned}
$$


Simplification of eq. (3.26) using eqs. (3.27), (3.28) yields

$$
\begin{aligned}
& x_{1} x_{2} s_{12}+x_{1} x_{3} s_{13}+x_{2} x_{3} s_{23}+x_{1} x_{4} s_{14}+x_{2} x_{4} s_{24}+x_{3} x_{4} s_{34} \\
& -m_{1}^{2} x_{1}-m_{2}^{2} x_{2}-m_{3}^{2} x_{3}-m_{4}^{2} x_{4}+m_{5}^{2}=0 .
\end{aligned}
$$

Solving the system of equations (3.27), (3.29) for $x_{1}, x_{4}$ we obtain

$$
x_{1}=\lambda_{4}, \quad x_{4}=1-x_{2}-x_{3}-\lambda_{4},
$$

where $\lambda_{4}$ is a solution of the equation

$$
A_{4} \lambda_{4}^{2}+B_{4} \lambda_{4}+C_{4}=0
$$

with

$$
\begin{aligned}
A_{4}= & s_{14}, \\
B_{4}= & \left(s_{24}-s_{12}+s_{14}\right) x_{2}+\left(s_{34}-s_{13}+s_{14}\right) x_{3}+m_{1}^{2}-m_{4}^{2}-s_{14}, \\
C_{4}= & s_{24} x_{2}^{2}+\left(s_{34}-s_{23}+s_{24}\right) x_{2} x_{3}+\left(m_{2}^{2}-m_{4}^{2}-s_{24}\right) x_{2}+s_{34} x_{3}^{2} \\
& +\left(m_{3}^{2}-m_{4}^{2}-s_{34}\right) x_{3}+m_{4}^{2}-m_{5}^{2} .
\end{aligned}
$$

Eqs. (3.4), (3.15) and (3.24) will be used in the next sections to derive functional equations for the propagator, vertex and box type of integrals.

\subsection{Algebraic relationship for products of any number of propagators}

The relations between the products of five and more propagators can be easily derived in the same way as it was done for the products of two-, three- and four-propagators. From eq. (3.2) one can derive a system of equations and find its solution for arbitrary $n$. Multiplying both sides of eq. (3.2) by the product $\prod_{j=1}^{n+1} P_{j}$ yields

$$
P_{n+1}=\sum_{r=1}^{n} x_{r} P_{r}
$$

or

$$
k_{1}^{2}-2 k_{1} p_{n+1}+p_{n+1}^{2}=\sum_{r=1}^{n} x_{r}\left(k_{1}^{2}-2 k_{1} p_{r}+p_{r}^{2}-m_{r}^{2}\right) .
$$

Again we assume that $k_{1}$ will be our integration momentum and $p_{j}, x_{r}$ do not depend on it. Differentiating eq. (3.34) with respect to $k_{1 \mu}$ one gets a linear equation in $k_{1}$ from which two equations follow

$$
\begin{aligned}
1 & =\sum_{r=1}^{n} x_{r}, \\
p_{n+1} & =\sum_{j=1}^{n} x_{j} p_{j} .
\end{aligned}
$$




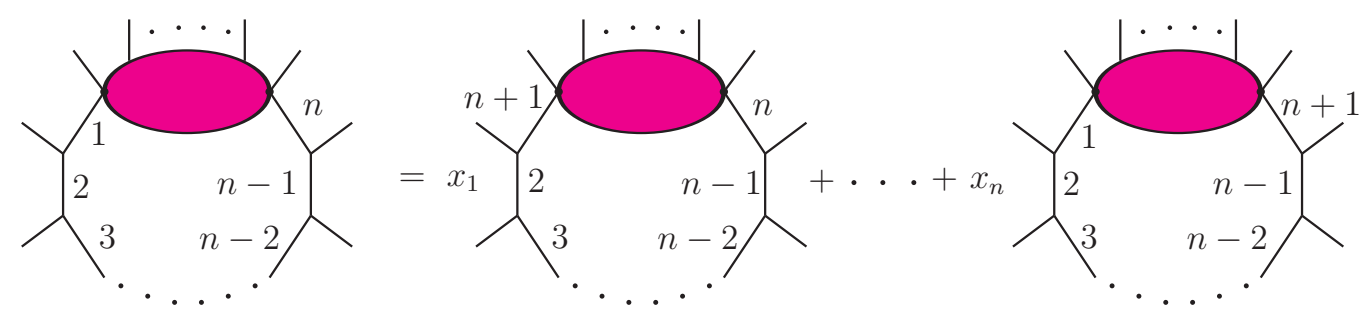

Figure 2. n-propagator functional equation.

Substituting eqs. (3.35), (3.36) into eq. (3.34) yields the following equation:

$$
m_{n+1}^{2}-\sum_{k=1}^{n} x_{k} m_{k}^{2}+\sum_{j=1}^{n} \sum_{l=1}^{j-1} x_{j} x_{l} s_{l j}=0 .
$$

Solving eq. (3.35) for one of the $x_{j}$ and substituting this solution into eq. (3.37) gives a quadratic equation for the remaining $x_{i}$. This quadratic equation can be solved with respect to one of the parameters $x_{j}$. Thus, the solution of the system of equations (3.35), (3.37) will depend on $n-2$ remaining arbitrary parameters $x_{i}$ and one arbitrary mass $m_{n}$.

It is interesting to note that for any $n$, the functional equations for integrals with all masses being equal to zero coinside with the functional equations for integrals with all equal masses and different from zero. In the case of equal masses, two mass dependent terms in eq. (3.37) cancel each other due to eq. (3.35). In both cases systems of equations for $x_{i}$ are the same and therefore arguments of integrals are the same.

Eq. (3.4) is analogous to the equation for splitting propagators presented in ref. [10]. Eq. (3.15) is a generalization of eq. (3.4). Indeed, setting $x_{3}=0$, canceling common factor $P_{3}$ on both sides of eq. (3.15) yields a relation similar to (3.4). In turn, eq. (3.24) is a generalization of eq. (3.15).

\subsection{Prototypes of functional equations}

Multiplying algebraic relations (3.4), (3.15), (3.24) by products of any number of propagators raised to arbitrary powers $\nu_{j}$

$$
\prod_{j=n_{0}}^{N} \frac{1}{\left[\left(k_{1}-p_{j}\right)-m_{j}^{2}\right]^{\nu_{j}}}
$$

and integrating with respect to $k_{1}$ we get a functional equation for one-loop integrals. Eqs. (3.4), (3.15), (3.24) can also be used to derive functional equations for integrals with any number of loops. Multiplying algebraic relations for propagators by the function corresponding to Feynman integral depending on momentum $k_{1}$ and any number of external momenta and then integrating with respect to $k_{1}$ will produce functional equations. Just for demonstrational purposes in figure 2 we present graphically a functional equation based on $n$ propagator relation. The blob on this picture corresponds to either a product of the propagators raised to arbitrary powers or an integral with any number of loops and external legs. Also, the blob can be a product of the propagators multiplied by an integral. One of the external momenta of this multiloop integral should be $k_{1}$. 


\section{Some examples of functional equations}

In this section several particular examples of the functional equations resulting from algebraic relations for the products of propagators will be considered.

\subsection{Functional equation for the one-loop propagator type integral}

First, we consider the simplest case, namely, a functional equation for the integral $I_{2}^{(d)}$ :

$$
I_{2}^{(d)}\left(m_{j}^{2}, m_{k}^{2} ; s_{j k}\right)=\int \frac{d^{d} k_{1}}{i \pi^{d / 2}} \frac{1}{\left[\left(k_{1}-p_{j}\right)^{2}-m_{j}^{2}\right]\left[\left(k_{1}-p_{k}\right)^{2}-m_{k}^{2}\right]} .
$$

Integrating both sides of eq. (3.4) with respect to $k_{1}$, we get:

$$
I_{2}^{(d)}\left(m_{1}^{2}, m_{2}^{2}, s_{12}\right)=x_{1} I_{2}^{(d)}\left(m_{2}^{2}, m_{3}^{2}, s_{23}\right)+x_{2} I_{2}^{(d)}\left(m_{1}^{2}, m_{3}^{2}, s_{13}\right) .
$$

The arguments $s_{13}, s_{23}$ of the integrals on the right-hand side depend on $x_{1}, x_{2}$

$$
\begin{aligned}
& s_{13}=\left(p_{1}-p_{3}\right)^{2}=\left(x_{1}-1\right)^{2} p_{1}^{2}+2 x_{2}\left(x_{1}-1\right) p_{1} p_{2}+x_{2}^{2} p_{2}^{2}, \\
& s_{23}=\left(p_{2}-p_{3}\right)^{2}=p_{1}^{2} x_{1}^{2}+2\left(x_{2}-1\right) x_{1} p_{1} p_{2}+\left(x_{2}-1\right)^{2} p_{2}^{2} .
\end{aligned}
$$

Substituting solution for $x_{j}$ from eq. (3.10) into eq. (4.3) yields:

$$
\begin{aligned}
& s_{13}=\frac{\Lambda_{2}+2 s_{12}\left(m_{1}^{2}+m_{3}^{2}\right)}{2 s_{12}} \pm \frac{m_{2}^{2}-m_{1}^{2}-s_{12}}{2 s_{12}} \sqrt{\Lambda_{2}+4 s_{12} m_{3}^{2}}, \\
& s_{23}=\frac{\Lambda_{2}+2 s_{12}\left(m_{2}^{2}+m_{3}^{2}\right)}{2 s_{12}} \pm \frac{m_{2}^{2}-m_{1}^{2}+s_{12}}{2 s_{12}} \sqrt{\Lambda_{2}+4 s_{12} m_{3}^{2}} .
\end{aligned}
$$

In these equations $m_{3}^{2}$ is an arbitrary parameter and it can be taken at will. The functional equation (4.2) is in agreement with the result presented in refs. [1, 2].

\subsection{Functional equations for the one-loop vertex type integral}

Functional equations for the vertex type integral

$$
\begin{aligned}
I_{3}^{(d)} & \left(m_{1}^{2}, m_{2}^{2}, m_{3}^{2}, s_{23}, s_{13}, s_{12}\right) \\
& =\int \frac{d^{d} k_{1}}{i \pi^{d / 2}} \frac{1}{\left[\left(k_{1}-p_{1}\right)^{2}-m_{1}^{2}\right]\left[\left(k_{1}-p_{2}\right)^{2}-m_{2}^{2}\right]\left[\left(k_{1}-p_{3}\right)^{2}-m_{3}^{2}\right]},
\end{aligned}
$$

can be obtained from eq. (3.4) as well as from eq. (3.15). Multiplying both sides of eq. (3.4) by the factor $1 / P_{4}$ where

$$
P_{4}=\left(k_{1}-p_{4}\right)^{2}-m_{4}^{2},
$$

and integrating over $k_{1}$ leads to the equation

$$
\int \frac{d^{d} k_{1}}{P_{1} P_{2} P_{4}}=x_{1} \int \frac{d^{d} k_{1}}{P_{2} P_{3} P_{4}}+x_{2} \int \frac{d^{d} k_{1}}{P_{1} P_{3} P_{4}} .
$$

In terms of integrals $I_{3}^{(d)}$ this equation reads

$$
\begin{aligned}
& I_{3}^{(d)}\left(m_{1}^{2}, m_{2}^{2}, m_{4}^{2} ; s_{24}, s_{14}, s_{12}\right) \\
& \quad=x_{1} I_{3}^{(d)}\left(m_{2}^{2}, m_{3}^{2}, m_{4}^{2} ; s_{34}, s_{24}, s_{23}\right)+x_{2} I_{3}^{(d)}\left(m_{1}^{2}, m_{3}^{2}, m_{4}^{2} ; s_{34}, s_{14}, s_{13}\right),
\end{aligned}
$$


where $s_{12}, s_{14}, s_{24}$ are independent scalar invariants, $s_{13}, s_{23}$ are given in eq. (4.4) and

$$
\begin{aligned}
s_{34}= & \frac{1}{2}\left(s_{14}+s_{24}-m_{1}^{2}-m_{2}^{2}+2 m_{3}^{2}\right) \\
& -\frac{\left(m_{1}^{2}-m_{2}^{2}-s_{14}+s_{24}\right)\left(m_{2}^{2}-m_{1}^{2} \pm \sqrt{\Lambda_{2}+4 m_{3}^{2}}\right)}{2 s_{12}} .
\end{aligned}
$$

Two more functional equations can be obtained from eq. (4.7) by symmetric permutations $\left(m_{1}, p_{1}\right) \leftrightarrow\left(m_{4}, p_{4}\right)$ and $\left(m_{2}, p_{2}\right) \leftrightarrow\left(m_{4}, p_{4}\right)$.

Another functional equation for the vertex type integral can be obtained by integrating both sides of eq. (3.15) with respect to $k_{1}$ :

$$
\begin{aligned}
I_{3}^{(d)}\left(m_{1}^{2},\right. & \left.m_{2}^{2}, m_{3}^{2}, s_{23}, s_{13}, s_{12}\right)=\left(1-\lambda_{3}-x_{3}\right) I_{3}^{(d)}\left(m_{4}^{2}, m_{2}^{2}, m_{3}^{2}, s_{23}, s_{34}, s_{24}\right) \\
& +\lambda_{3} I_{3}^{(d)}\left(m_{1}^{2}, m_{4}^{2}, m_{3}^{2}, s_{34}, s_{13}, s_{14}\right)+x_{3} I_{3}^{(d)}\left(m_{1}^{2}, m_{2}^{2}, m_{4}^{2}, s_{24}, s_{14}, s_{12}\right),
\end{aligned}
$$

where $s_{12}, s_{13}, s_{23}$ are arbitrary scalar invariants and

$$
\begin{aligned}
& s_{14}=\left(m_{1}^{2}-m_{2}^{2}+s_{12}\right) \lambda_{3}+\left(m_{1}^{2}-m_{3}^{2}+s_{13}\right) x_{3}+m_{4}^{2}-m_{1}^{2}, \\
& s_{24}=\left(m_{1}^{2}-s_{12}-m_{2}^{2}\right) \lambda_{3}+\left(m_{1}^{2}-m_{3}^{2}-s_{12}+s_{23}\right) x_{3}+m_{4}^{2}-m_{1}^{2}+s_{12}, \\
& s_{34}=\left(m_{1}^{2}-s_{13}+s_{23}-m_{2}^{2}\right) \lambda_{3}+\left(m_{1}^{2}-m_{3}^{2}-s_{13}\right) x_{3}+m_{4}^{2}-m_{1}^{2}+s_{13} .
\end{aligned}
$$

There is an essential difference between the functional equation eq. (4.8) obtained from eq. (3.4) and the functional equation (4.10) derived from eq. (3.15). For example, at $m_{1}^{2}=m_{2}^{2}=m_{3}^{2}=m_{4}^{2}=0$, eq. (4.8) becomes trivial while from eq. (4.10) for the integral

$$
I_{3}^{(d)}\left(s_{23}, s_{13}, s_{12}\right) \equiv I_{3}^{(d)}\left(0,0,0, s_{23}, s_{13}, s_{12}\right)
$$

we obtain a nontrivial functional equation

$$
\begin{aligned}
I_{3}^{(d)}( & \left.s_{23}, s_{13}, s_{12}\right) \\
= & \left(1-\lambda_{3}-x_{3}\right) I_{3}^{(d)}\left(s_{12}\left(1-\lambda_{3}-x_{3}\right)+s_{23} x_{3}, s_{23}, \lambda_{3}\left(s_{23}-s_{13}\right)+s_{13}\left(1-x_{3}\right)\right) \\
& +\lambda_{3} I_{3}^{(d)}\left(s_{13} x_{3}+\lambda_{3} s_{12}, \lambda_{3}\left(s_{23}-s_{13}\right)+s_{13}\left(1-x_{3}\right), s_{13}\right) \\
& +x_{3} I_{3}^{(d)}\left(s_{12}, s_{12}\left(1-\lambda_{3}-x_{3}\right)+s_{23} x_{3}, s_{13} x_{3}+\lambda_{3} s_{12}\right),
\end{aligned}
$$

where $\lambda_{3}$ is a root of the quadratic equation

$$
s_{12} \lambda_{3}^{2}-\left(s_{12}-s_{12} x_{3}-s_{13} x_{3}+s_{23} x_{3}\right) \lambda_{3}+x_{3}^{2} s_{13}-x_{3} s_{13}=0 .
$$

If one argument of $I_{3}^{(d)}\left(s_{23}, s_{13}, s_{12}\right)$ is zero, then by applying functional equation (4.13) such an integral can be expressed in terms of integrals $I_{3}^{(d)}$ with two arguments equal to zero. For example, at $s_{23}=0$ and $x_{3}=s_{12} /\left(s_{12}-s_{13}\right)$ the relation (4.13) becomes

$$
I_{3}^{(d)}\left(0, s_{13}, s_{12}\right)=\frac{s_{12}}{s_{12}-s_{13}} I_{3}^{(d)}\left(s_{12}, 0,0\right)-\frac{s_{13}}{s_{12}-s_{13}} I_{3}^{(d)}\left(0,0, s_{13}\right) .
$$

This is a typical example how functional equations can be used to simplify evaluation of an integral by reducing it to a combination of integrals with fewer arguments. 
At $m_{1}^{2}=m_{2}^{2}=m_{3}^{2}=m_{4}^{2}=m^{2}$, similar to the previous case, eq. (4.8) degenerate while from eq. (4.10) for the integral

$$
I_{3}^{(d)}\left(m^{2}, m^{2}, m^{2}, s_{23}, s_{13}, s_{12}\right) \equiv I_{3}^{(d)}\left(m^{2} ; s_{23}, s_{13}, s_{12}\right)
$$

we obtain a nontrivial functional equation

$$
\begin{aligned}
I_{3}^{(d)}( & \left.m^{2} ; s_{23}, s_{13}, s_{12}\right) \\
= & \left(1-\lambda_{3}-x_{3}\right) I_{3}^{(d)}\left(m^{2} ; s_{12}\left(1-\lambda_{3}-x_{3}\right)+s_{23} x_{3}, s_{23}, \lambda_{3}\left(s_{23}-s_{13}\right)+s_{13}\left(1-x_{3}\right)\right) \\
& +\lambda_{3} I_{3}^{(d)}\left(m^{2} ; s_{13} x_{3}+\lambda_{3} s_{12}, \lambda_{3}\left(s_{23}-s_{13}\right)+s_{13}\left(1-x_{3}\right), s_{13}\right) \\
& +x_{3} I_{3}^{(d)}\left(m^{2} ; s_{12}, s_{12}\left(1-\lambda_{3}-x_{3}\right)+s_{23} x_{3}, s_{13} x_{3}+\lambda_{3} s_{12}\right)
\end{aligned}
$$

where $\lambda_{3}$ is a root of the quadratic equation

$$
s_{12} \lambda_{3}^{2}-\left(s_{12}-s_{12} x_{3}-s_{13} x_{3}+s_{23} x_{3}\right) \lambda_{3}+x_{3}^{2} s_{13}-x_{3} s_{13}=0 .
$$

Eqs. (4.17), (4.18) are identical to eqs. (4.13), (4.14) respectively and therefore the functional equation for the integral with massless propagators and the functional equation for the integral with all masses equal are the same. Setting $s_{23}=0$ and $x_{3}=s_{12} /\left(s_{12}-s_{13}\right)$ in eq. (4.17) leads to the relation similar to (4.15):

$$
I_{3}^{(d)}\left(m^{2} ; 0, s_{13}, s_{12}\right)=\frac{s_{12}}{s_{12}-s_{13}} I_{3}^{(d)}\left(m^{2} ; s_{12}, 0,0\right)-\frac{s_{13}}{s_{12}-s_{13}} I_{3}^{(d)}\left(m^{2} ; 0,0, s_{13}\right) .
$$

This is not surprising because the coefficients of the eq. (4.17) are mass independent and in the integrand $m^{2}$ and $i \eta$ appear in the covariant combination $m^{2}-i \eta$. For this reason, the similarity of functional equations for massless integrals and integrals with all masses equal take place for integrals with more external legs and more loops.

\subsection{Functional equations for one-loop box type integrals}

Functional equations for the box type integrals can be obtained by multiplying relation (3.4) by two propagators, or by multiplying relation (3.15) by one propagator and then integrating over momentum $k_{1}$. Yet another relation can be obtained just by integrating eq. (3.24) over momentum $k_{1}$ :

$$
\begin{aligned}
I_{4}^{(d)}( & \left.m_{1}^{2}, m_{2}^{2}, m_{3}^{2}, m_{4}^{2} ; s_{12}, s_{23}, s_{34}, s_{14}, s_{24}, s_{13}\right) \\
= & \lambda_{4} I_{4}^{(d)}\left(m_{5}^{2}, m_{2}^{2}, m_{3}^{2}, m_{4}^{2} ; s_{25}, s_{23}, s_{34}, s_{45}, s_{24}, s_{35}\right) \\
& \quad+x_{2} I_{4}^{(d)}\left(m_{1}^{2}, m_{5}^{2}, m_{3}^{2}, m_{4}^{2} ; s_{15}, s_{35}, s_{34}, s_{14}, s_{45}, s_{13}\right) \\
& +x_{3} I_{4}^{(d)}\left(m_{1}^{2}, m_{2}^{2}, m_{5}^{2}, m_{4}^{2} ; s_{12}, s_{25}, s_{45}, s_{14}, s_{24}, s_{15}\right) \\
& +\left(1-x_{2}-x_{3}-\lambda_{4}\right) I_{4}^{(d)}\left(m_{1}^{2}, m_{2}^{2}, m_{3}^{2}, m_{5}^{2} ; s_{12}, s_{23}, s_{35}, s_{15}, s_{25}, s_{13}\right) .
\end{aligned}
$$




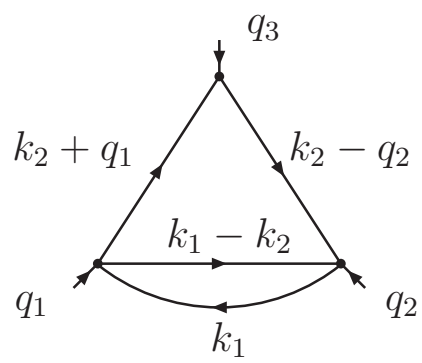

Figure 3. Diagram corresponding to $R\left(m_{1}^{2}, m_{2}^{2}, m_{3}^{2}, m_{4}^{2} ; q_{1}^{2}, q_{2}^{2}, q_{3}^{2}\right)$.

Here $\lambda_{4}$ is defined in eq. (3.31) and $m_{5}, x_{2}, x_{3}$ are arbitrary parameters and

$$
\begin{aligned}
s_{15}= & \lambda_{4}\left(m_{4}^{2}-s_{14}-m_{1}^{2}\right)+x_{3}\left(m_{4}^{2}-m_{3}^{2}-s_{14}+s_{13}\right)+x_{2}\left(m_{4}^{2}-m_{2}^{2}-s_{14}+s_{12}\right) \\
& +s_{14}+m_{5}^{2}-m_{4}^{2}, \\
s_{25}= & \lambda_{4}\left(m_{4}^{2}-m_{1}^{2}-s_{24}+s_{12}\right)+x_{3}\left(m_{4}^{2}-m_{3}^{2}-s_{24}+s_{23}\right)+x_{2}\left(m_{4}^{2}-m_{2}^{2}-s_{24}\right) \\
& +s_{24}+m_{5}^{2}-m_{4}^{2}, \\
s_{35}= & \lambda_{4}\left(m_{4}^{2}-m_{1}^{2}-s_{34}+s_{13}\right)+x_{3}\left(m_{4}^{2}-m_{3}^{2}-s_{34}\right)+x_{2}\left(m_{4}^{2}-m_{2}^{2}-s_{34}+s_{23}\right) \\
& +s_{34}+m_{5}^{2}-m_{4}^{2}, \\
s_{45}= & \lambda_{4}\left(s_{14}+m_{4}^{2}-m_{1}^{2}\right)+x_{3}\left(s_{34}+m_{4}^{2}-m_{3}^{2}\right)+x_{2}\left(s_{24}+m_{4}^{2}-m_{2}^{2}\right) \\
& +m_{5}^{2}-m_{4}^{2} .
\end{aligned}
$$

In this functional equation the arbitrary parameters can be chosen from the requirement of simplicity of evaluation of integrals on the right-hand side of eq. (4.20) or from some other requirements. For example, one can choose these parameters by transforming arguments to a certain kinematical region needed for the analytic continuation of the original integral.

\subsection{Functional equation for two-loop vertex type integral}

The method described in the previous section can be applied to multiloop integrals. Consider, for example, an integral corresponding to the diagram given in figure 3 . If we multiply eq. (3.4) by the one-loop integral depending on $k_{1}$

$$
\int \frac{d^{d} k_{2}}{\left[k_{2}^{2}-m_{4}^{2}\right]\left[\left(k_{1}-k_{2}\right)^{2}-m_{5}^{2}\right]}
$$

and integrate with respect to momentum $k_{1}$ then we obtain the functional equation

$$
\begin{aligned}
& R\left(m_{1}^{2}, m_{2}^{2}, m_{3}^{2}, m_{4}^{2} ; q_{1}^{2}, q_{2}^{2}, q_{3}^{2}\right) \\
& \quad=\alpha R\left(0, m_{2}^{2}, m_{3}^{2}, m_{4}^{2} ; Q^{2}, q_{2}^{2},\left(m_{2}^{2}-m_{1}^{2}+q_{3}^{2}\right) \alpha-m_{2}^{2}\right) \\
& \quad+(1-\alpha) R\left(m_{1}^{2}, 0, m_{3}^{2}, m_{4}^{2} ; q_{1}^{2}, Q^{2},\left(m_{2}^{2}-m_{1}^{2}-q_{3}^{2}\right) \alpha+q_{3}^{2}-m_{2}^{2}\right),
\end{aligned}
$$


where

$$
\begin{aligned}
R & \left.m_{1}^{2}, m_{2}^{2}, m_{3}^{2}, m_{4}^{2} ; q_{1}^{2}, q_{2}^{2}, q_{3}^{2}\right) \\
& =\iint \frac{d^{d} k_{1} d^{d} k_{2}}{\left(i \pi^{d / 2}\right)^{2}} \frac{1}{\left[\left(k_{2}+q_{1}\right)^{2}-m_{1}^{2}\right]\left[\left(k_{2}-q_{2}\right)^{2}-m_{2}^{2}\right]\left[k_{1}^{2}-m_{3}^{2}\right]\left[\left(k_{1}-k_{2}\right)^{2}-m_{4}^{2}\right]}, \\
Q^{2} & =\left(q_{1}^{2}-q_{2}^{2}-m_{1}^{2}+m_{2}^{2}\right) \alpha+q_{2}^{2}-m_{2}^{2}, \\
\alpha & =\frac{q_{3}^{2}-m_{1}^{2}+m_{2}^{2} \pm \sqrt{\Delta}}{2 q_{3}^{2}} \\
\Delta & =q_{3}^{4}+m_{1}^{4}+m_{2}^{4}-2 q_{3}^{2} m_{1}^{2}-2 q_{3}^{2} m_{2}^{2}-2 m_{1}^{2} m_{2}^{2} .
\end{aligned}
$$

Integrals of this type arise, for example, in calculations of two-loop radiative corrections in the electroweak theory. Instead of the integral $R\left(m_{1}^{2}, m_{2}^{2}, m_{3}^{2}, m_{4}^{2} ; q_{1}^{2}, q_{2}^{2}, q_{3}^{2}\right)$, one can consider a derivative of $R$ with respect to $m_{3}^{2}$ which is UV finite:

$$
\begin{aligned}
\frac{\partial R}{\partial m_{3}^{2}} & \equiv R_{3}\left(m_{1}^{2}, m_{2}^{2}, m_{3}^{2}, m_{4}^{2} ; q_{1}^{2}, q_{2}^{2}, q_{3}^{2}\right) \\
& =\iint \frac{d^{d} k_{1} d^{d} k_{2}}{\left(i \pi^{d / 2}\right)^{2}} \frac{1}{\left[\left(k_{2}+q_{1}\right)^{2}-m_{1}^{2}\right]\left[\left(k_{2}-q_{2}\right)^{2}-m_{2}^{2}\right]\left[k_{1}^{2}-m_{3}^{2}\right]^{2}\left[\left(k_{1}-k_{2}\right)^{2}-m_{4}^{2}\right]}
\end{aligned}
$$

Integral $R_{3}$ satisfies the following functional equations:

$$
\begin{aligned}
& R_{3}\left(m_{1}^{2}, m_{2}^{2}, m_{3}^{2}, m_{4}^{2} ; q_{1}^{2}, q_{2}^{2}, q_{3}^{2}\right) \\
& \quad=\alpha R_{3}\left(0, m_{2}^{2}, m_{3}^{2}, m_{4}^{2} ; Q^{2}, q_{2}^{2},\left(m_{2}^{2}-m_{1}^{2}+q_{3}^{2}\right) \alpha-m_{2}^{2}\right) \\
& \quad+(1-\alpha) R_{3}\left(m_{1}^{2}, 0, m_{3}^{2}, m_{4}^{2} ; q_{1}^{2}, Q^{2},\left(m_{2}^{2}-m_{1}^{2}-q_{3}^{2}\right) \alpha+q_{3}^{2}-m_{2}^{2}\right) .
\end{aligned}
$$

This relation can be used for computing the basis integral arising in calculation of the two-loop radiative correction to the ortho-positronium lifetime. In particular, one of these basis integrals corresponds to kinematics $m_{1}^{2}=m_{2}^{2}=m_{3}^{2}=m_{4}^{2}=m^{2}, q_{1}^{2}=q_{2}^{2}=m^{2}$, $q_{3}^{2}=4 m^{2}$. In this case relation (4.29) reads

$$
R_{3}\left(m^{2}, m^{2}, m^{2}, m^{2} ; m^{2}, m^{2}, 4 m^{2}\right)=R_{3}\left(0, m^{2}, m^{2}, m^{2} ; 0, m^{2}, m^{2}\right) .
$$

In fact the integral on the right-hand side is a propagator type integral with one massless line. Applying the recurrence relations given in ref. [11], this integral can be reduced to a simpler integral:

$$
\begin{aligned}
R_{3} & \left(0, m^{2}, m^{2}, m^{2} ; 0, m^{2}, m^{2}\right) \\
& =\frac{1}{\left(i \pi^{d / 2}\right)^{2}} \iint \frac{d^{d} k_{1} d^{d} k_{2}}{k_{1}^{2}\left(k_{2}^{2}-m^{2}\right)\left[\left(k_{1}-k_{2}\right)^{2}-m^{2}\right]^{2}\left[\left(k_{1}+q_{1}\right)^{2}-m^{2}\right]} \\
& =\frac{2}{3(d-3)} J_{111}^{(d-2)}\left(m^{2}\right),
\end{aligned}
$$

where

$$
J_{111}^{(d)}\left(q^{2}\right)=\frac{1}{\left(i \pi^{d / 2}\right)^{2}} \iint \frac{d^{d} k_{1} d^{d} k_{2}}{\left(k_{1}^{2}-m^{2}\right)\left[\left(k_{1}-k_{2}\right)^{2}-m^{2}\right]\left[\left(k_{2}-q\right)^{2}-m^{2}\right]} .
$$




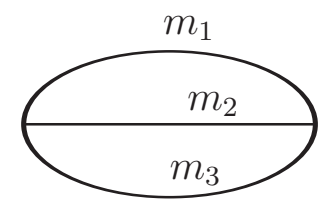

Figure 4. Two-loop vacuum type diagram.

At $q^{2}=m^{2}$, the result for $J_{111}^{(d)}\left(q^{2}\right)$ is known [12]:

$$
\begin{aligned}
& \frac{m^{6-2 d}}{8}(d-4)^{2}(d-2)(d-3) J_{111}^{(d)}\left(m^{2}\right) \\
& \quad={ }_{3} F_{2}\left[\begin{array}{c}
1,3-d, \frac{1}{2} ; \\
\frac{5-d}{2}, \frac{d}{2} ;
\end{array}\right]+(d-3){ }_{3} F_{2}\left[\begin{array}{c}
1, \frac{4-d}{2}, \frac{d-1}{2} ; 1 \\
\frac{3}{2}, d-1 ;
\end{array}\right],
\end{aligned}
$$

and it can be used for the $\varepsilon$ expansion of $R$ and $R_{3}$. As was already mentioned, at $q_{1}^{2}=q_{2}^{2}=m^{2}, q_{3}^{2}=4 m^{2}$ integrals on the right-hand side of eq. (4.23) correspond to the propagator type integrals. Analytic result for $R$ reads

$$
\begin{aligned}
& (d-3) R\left(m^{2}, m^{2}, m^{2}, m^{2}, m^{2}, m^{2}, 4 m^{2}\right) \\
& \quad=\frac{1}{8 m^{4}(d-4)}\left[2 m^{2}(3 d-8)(d-3) J_{111}^{(d)}-3(d-2)^{2}\left(T_{1}^{(d)}\right)^{2}\right],
\end{aligned}
$$

where

$$
T_{1}^{(d)}=-\Gamma\left(1-\frac{d}{2}\right)\left(m^{2}\right)^{(d-2) / 2} .
$$

We checked that the first few terms in the $\varepsilon=(4-d) / 2$ expansion of $R$ and $R_{3}$ are in agreement with results of [13]. The main profit from the functional equations for $R$ and $R_{3}$ comes from the fact that vertex integrals were expressed in terms of simpler, propagator type integrals.

\section{Deriving functional equation by deforming propagators}

The method described in the previous section does not work for deriving functional equations for all kinds of Feynman integrals. For example, we did not find a functional equation for the two-loop vacuum type integral given in figure 4 . In this section we shall describe another method that extends the class of integrals for which we can obtain functional equations. The method is based on transformation of functional equations for some auxiliary integrals depending on arbitrary parameters into functional equations for integrals of interest. Such functional equations will be derived from algebraic relations for 'deformed propagators' which will be defined in the next section. These auxiliary integrals will be transformed into $\alpha$ parametric representation. In general characteristic polynomials of these integrals in $\alpha$ parametric representation differ from those for the investigated integral. The functional equation for the integral of interest can be obtained in case when it is possible to map characteristic polynomials of auxiliary integrals with 'deformed propagators' to characteristic polynomials of this integral. Such a mapping will be performed by rescaling $\alpha$ parameters and appropriate choice of arbitrary 'deforming parameters'. 


\subsection{Algebraic relations for products of deformed propagators}

In the previous section, in order to derive the functional equation, we added to our consideration a propagator with combination of external momenta taken with arbitrary scalar coefficients. Now we consider a generalization of this method.

To find functional equation for $L$-loop Feynman integral depending on $E$-external momenta, we start from the relation of the form

$$
\prod_{r=1}^{n} \frac{1}{D_{r}}=\frac{1}{D_{n+1}} \sum_{r=1}^{n} x_{r} \prod_{\substack{j=1 \\ j \neq r}}^{n} \frac{1}{D_{j}}
$$

where $D_{j}$ is defined as

$$
D_{j}=Q_{j}^{2}-m_{j}^{2}+i \eta
$$

with

$$
Q_{j}=\sum_{l=1}^{L} a_{j l}^{(j)} k_{l}+\sum_{l=1}^{E+1} b_{j l}^{(j)} p_{l}
$$

and $a_{j l}^{(j)}, b_{j l}^{(j)}$ for the time being are arbitrary scalar parameters. Some of these parameters as well as $x_{r}$ will be fixed from eq. (5.1). Another part of these parameters will be fixed from the requirement that the product of propagators in eq. (5.1) should correspond to the integrand of the integral with the considered topology. We would like to remark that instead of the deformation of the propagators proposed in eqs. (5.2), (5.3), one can use other deformations. For example, all terms in denominators of propagators can be taken with arbitrary scalar coefficients:

$$
Q_{j}^{2}=\sum_{l, r=1}^{L} a_{r l}^{(j)}\left(k_{r} \cdot k_{l}\right)+\sum_{l=1}^{L} \sum_{r=1}^{E+1} b_{r l}^{(j)}\left(p_{r} \cdot k_{l}\right)+\sum_{r, l=1}^{E+1} c_{r l}^{(j)}\left(p_{r} \cdot p_{l}\right) .
$$

To establish algebraic relation (5.1), we put all terms over a common denominator and then equate coefficients in front of scalar products depending on integration momenta. Solving the obtained system of equations gives some restrictions on the scalar parameters.

In general, the integrals obtained by integrating products of 'deformed propagators' will not correspond to usual Feynman integrals. Further restrictions on parameters should be imposed in order to obtain relations between the integrals corresponding to Feynman integrals coming from a realistic quantum field theory models.

\subsection{Functional equation for two-loop vacuum type integral with arbitrary masses}

As an example, let us consider a derivation of the functional equation for the two-loop vacuum type integral given in figure 4 :

$$
J_{0}^{(d)}\left(m_{1}^{2}, m_{2}^{2}, m_{3}^{2}\right)=\iint \frac{\mathrm{d}^{d} k_{1} \mathrm{~d}^{d} k_{2}}{\left(i \pi^{d / 2}\right)^{2}} \frac{1}{\left(k_{1}^{2}-m_{1}^{2}\right)\left(\left(k_{1}-k_{2}\right)^{2}-m_{2}^{2}\right)\left(k_{2}^{2}-m_{3}^{2}\right)} .
$$


An analytic expression for this integral was presented in ref. [14]. Instead of this integral we will first consider an auxiliary integral with the integrand made from the 'deformed propagators' defined in eqs. (5.2), (5.3):

$$
\widetilde{J}_{0}^{(d)}\left(m_{1}^{2}, m_{2}^{2}, m_{3}^{2}\right)=\iint \frac{\mathrm{d}^{d} k_{1} \mathrm{~d}^{d} k_{2}}{\left(i \pi^{d / 2}\right)^{2}} \frac{1}{D_{1} D_{2} D_{3}},
$$

where

$$
\begin{aligned}
& D_{1}=\left(a_{1} k_{1}+a_{2} k_{2}\right)^{2}-m_{1}^{2}+i \eta, \quad D_{2}=\left(b_{1} k_{1}+b_{2} k_{2}\right)^{2}-m_{2}^{2}+i \eta, \\
& D_{3}=\left(h_{1} k_{1}+h_{2} k_{2}\right)^{2}-m_{3}^{2}+i \eta .
\end{aligned}
$$

For the product of three deformed propagators one can try to find an algebraic relation of the form

$$
\frac{1}{D_{1} D_{2} D_{3}}=\frac{x_{1}}{D_{4} D_{2} D_{3}}+\frac{x_{2}}{D_{1} D_{4} D_{3}}+\frac{x_{3}}{D_{1} D_{2} D_{4}},
$$

where $D_{1}, D_{2}, D_{3}$ are defined in eq. (5.7) and

$$
D_{4}=\left(r_{1} k_{1}+r_{2} k_{2}\right)^{2}-m_{4}^{2}+i \eta
$$

Here $m_{k}$ are arbitrary masses, $x_{k}, a_{j}, b_{i}, h_{s}, r_{l}$ are undetermined parameters and $k_{1}, k_{2}$ will be integration momenta.

Putting in eq. (5.8) all over a common denominator and equating to zero coefficients in front of different products of $k_{1}^{2}, k_{2}^{2}$ and $k_{1} k_{2}$ leads to the system of equations:

$$
\begin{array}{rrr}
r_{1}^{2}-x_{1} a_{1}^{2}-x_{2} b_{1}^{2}-x_{3} h_{1}^{2}=0, & r_{1} r_{2}-x_{1} a_{1} a_{2}-x_{2} b_{1} b_{2}-x_{3} h_{1} h_{2}=0, \\
r_{2}^{2}-x_{1} a_{2}^{2}-x_{2} b_{2}^{2}-x_{3} h_{2}^{2}=0, & m_{4}^{2}-x_{1} m_{1}^{2}-x_{2} m_{2}^{2}-x_{3} m_{3}^{2}=0 .
\end{array}
$$

Solving this system for $r_{1}, x_{1}, x_{2}, x_{3}$ we have:

$$
\begin{aligned}
r_{1} & =r_{2} \lambda \\
A x_{1} & =r_{2}^{2}\left(h_{1} h_{2} m_{2}^{2}-b_{2} b_{1} m_{3}^{2}\right)+b_{2} h_{2}\left(b_{1} h_{2}-b_{2} h_{1}\right) m_{4}^{2}-r_{2}^{2}\left(h_{2}^{2} m_{2}^{2}-m_{3}^{2} b_{2}^{2}\right) \lambda, \\
A x_{2} & =-r_{2}^{2}\left(m_{1}^{2} h_{2} h_{1}-a_{2} a_{1} m_{3}^{2}\right)-a_{2} h_{2}\left(a_{1} h_{2}-a_{2} h_{1}\right) m_{4}^{2}+r_{2}^{2}\left(h_{2}^{2} m_{1}^{2}-m_{3}^{2} a_{2}^{2}\right) \lambda, \\
A x_{3} & =r_{2}^{2}\left(m_{1}^{2} b_{2} b_{1}-a_{2} a_{1} m_{2}^{2}\right)+a_{2} b_{2}\left(a_{1} b_{2}-a_{2} b_{1}\right) m_{4}^{2}-r_{2}^{2}\left(b_{2}^{2} m_{1}^{2}-m_{2}^{2} a_{2}^{2}\right) \lambda
\end{aligned}
$$

where $\lambda$ is a root of the quadratic equation

$$
A \lambda^{2}+B \lambda+C=0
$$

and

$$
\begin{aligned}
A= & b_{2} h_{2}\left(b_{1} h_{2}-h_{1} b_{2}\right) m_{1}^{2}+a_{2} h_{2}\left(h_{1} a_{2}-a_{1} h_{2}\right) m_{2}^{2}+a_{2} b_{2}\left(a_{1} b_{2}-b_{1} a_{2}\right) m_{3}^{2}, \\
B= & \left(h_{1} b_{2}-b_{1} h_{2}\right)\left(b_{1} h_{2}+h_{1} b_{2}\right) m_{1}^{2}+\left(a_{1} h_{2}-h_{1} a_{2}\right)\left(a_{1} h_{2}+h_{1} a_{2}\right) m_{2}^{2} \\
& +\left(b_{1} a_{2}-a_{1} b_{2}\right)\left(a_{1} b_{2}+b_{1} a_{2}\right) m_{3}^{2}, \\
C= & b_{1} h_{1}\left(b_{1} h_{2}-h_{1} b_{2}\right) m_{1}^{2}+a_{1} h_{1}\left(a_{2} h_{1}-a_{1} h_{2}\right) m_{2}^{2}+a_{1} b_{1}\left(a_{1} b_{2}-b_{1} a_{2}\right) m_{3}^{2} \\
& +\left(b_{2} h_{1}-b_{1} h_{2}\right)\left(a_{1} h_{2}-a_{2} h_{1}\right)\left(a_{1} b_{2}-a_{2} b_{1}\right) m_{4}^{2} .
\end{aligned}
$$


In order to obtain functional equation for the integral $J_{0}^{(d)}$, we integrate first both sides of the eq. (5.8) with respect to $k_{1}, k_{2}$ and then convert these integrals into the $\alpha$-parametric representation. Transforming all propagators into a parametric form

$$
\frac{1}{\left(k^{2}-m^{2}+i \eta\right)^{\nu}}=\frac{i^{-\nu}}{\Gamma(\nu)} \int_{0}^{\infty} d \alpha \alpha^{\nu-1} \exp \left[i \alpha\left(k^{2}-m^{2}+i \eta\right)\right]
$$

and using the $d$-dimensional Gaussian integration formula

$$
\int d^{d} k \exp \left[i\left(a k^{2}+2(p k)\right)\right]=i\left(\frac{\pi}{i a}\right)^{\frac{d}{2}} \exp \left[-\frac{i p^{2}}{a}\right]
$$

we can easily evaluate the integrals over loop momenta. The final result is:

$$
\widetilde{J}_{0}^{(d)}\left(m_{1}^{2}, m_{2}^{2}, m_{3}^{2}\right)=i^{1-d} \int_{0}^{\infty} \int_{0}^{\infty} \int_{0}^{\infty} \frac{d \alpha_{1} d \alpha_{2} d \alpha_{3}}{[\widetilde{D}(\alpha)]^{\frac{d}{2}}} \exp \left[-i \sum_{l=1}^{3} \alpha_{l}\left(m_{l}^{2}-i \eta\right)\right],
$$

where the polynomial

$$
\widetilde{D}(\alpha)=\left(a_{1} b_{2}-a_{2} b_{1}\right)^{2} \alpha_{1} \alpha_{2}+\left(a_{1} h_{2}-a_{2} h_{1}\right)^{2} \alpha_{1} \alpha_{3}+\left(b_{1} h_{2}-b_{2} h_{1}\right)^{2} \alpha_{2} \alpha_{3},
$$

differs from the appropriate $D$ polynomial of the two-loop vacuum integral defined in eq. (5.5)

$$
D(\alpha)=\alpha_{1} \alpha_{2}+\alpha_{1} \alpha_{3}+\alpha_{2} \alpha_{3}
$$

Changing in eq. (5.16) integration variables

$$
\alpha_{j} \rightarrow \alpha_{j} \theta_{j}^{2}
$$

with

$$
\theta_{1}=b_{1} h_{2}-b_{2} h_{1}, \quad \theta_{2}=a_{1} h_{2}-a_{2} h_{1}, \quad \theta_{3}=a_{1} b_{2}-a_{2} b_{1},
$$

leads to the relation:

$$
\widetilde{D}=\left(b_{1} h_{2}-b_{2} h_{1}\right)^{2}\left(a_{1} h_{2}-a_{2} h_{1}\right)^{2}\left(a_{1} b_{2}-a_{2} b_{1}\right)^{2}\left(\alpha_{1} \alpha_{2}+\alpha_{1} \alpha_{3}+\alpha_{2} \alpha_{3}\right) .
$$

Therefore integral $\widetilde{J}_{0}^{(d)}$ with deformed propagators is proportional to $J_{0}^{(d)}$ with modified arguments:

$$
\begin{aligned}
\iint \frac{d^{d} k_{1} d^{d} k_{2}}{\left(i \pi^{d / 2}\right)^{2}} \frac{1}{D_{1} D_{2} D_{3}} & =i^{1-d}\left[\theta_{1}^{2} \theta_{2}^{2} \theta_{3}^{2}\right]^{\frac{2-d}{2}} \int_{0}^{\infty} \int_{0}^{\infty} \int_{0}^{\infty} \frac{d \alpha_{1} d \alpha_{2} d \alpha_{3}}{D^{\frac{d}{2}}} \exp [-i \mathcal{M}] \\
& =\left[\theta_{1}^{2} \theta_{2}^{2} \theta_{3}^{2}\right]^{\frac{2-d}{2}} J_{0}^{(d)}\left(\theta_{1}^{2} m_{1}^{2}, \theta_{2}^{2} m_{2}^{2}, \theta_{3}^{2} m_{3}^{2}\right)
\end{aligned}
$$

where

$$
\mathcal{M}=\alpha_{1} \theta_{1}^{2} m_{1}^{2}+\alpha_{2} \theta_{2}^{2} m_{2}^{2}+\alpha_{3} \theta_{3}^{2} m_{3}^{2}
$$


With the aid of eq. (5.22), the relation (5.8) integrated with respect to $k_{1}, k_{2}$ can be written as a combination of integrals $J_{0}^{(d)}$ with different arguments:

$$
\begin{aligned}
& {\left[\theta_{1}^{2} \theta_{2}^{2} \theta_{3}^{2}\right]^{\frac{2-d}{2}} J_{0}^{(d)}\left(\theta_{1}^{2} m_{1}^{2}, \theta_{2}^{2} m_{2}^{2}, \theta_{3}^{2} m_{3}^{2}\right)} \\
& =x_{1}\left[\theta_{1}^{2} \theta_{6}^{2} \theta_{4}^{2}\right]^{2-d} J_{0}^{(d)}\left(\theta_{1}^{2} m_{4}^{2}, \theta_{6}^{2} m_{2}^{2}, \theta_{4}^{2} m_{3}^{2}\right) \\
& \quad+x_{2}\left[\theta_{2}^{2} \theta_{5}^{2} \theta_{6}^{2}\right]^{\frac{2-d}{2}} J_{0}^{(d)}\left(\theta_{6}^{2} m_{1}^{2}, \theta_{2}^{2} m_{4}^{2}, \theta_{5}^{2} m_{3}^{2}\right) \\
& \quad+x_{3}\left[\theta_{3}^{2} \theta_{4}^{2} \theta_{5}^{2}\right]^{\frac{2-d}{2}} J_{0}^{(d)}\left(\theta_{4}^{2} m_{1}^{2}, \theta_{5}^{2} m_{2}^{2}, \theta_{3}^{2} m_{4}^{2}\right)
\end{aligned}
$$

where $\theta_{1}, \theta_{2}, \theta_{3}$ are defined in (5.20) and

$$
\theta_{4}=r_{1} b_{2}-r_{2} b_{1}, \quad \theta_{5}=r_{1} a_{2}-r_{2} a_{1}, \quad \theta_{6}=r_{1} h_{2}-r_{2} h_{1}
$$

Now we consider relation (5.24) as an equation for integrals in momentum representation. By rescaling integration variables $k_{1}, k_{2}$ in the integral on the left-hand side

$$
k_{1}=\left(\theta_{1} \theta_{2} \theta_{3}\right)^{\frac{1}{2}} \widetilde{k}_{1}, \quad k_{2}=\left(\theta_{1} \theta_{2} \theta_{3}\right)^{\frac{1}{2}} \widetilde{k}_{2}
$$

and performing analogous changes for the integrals on the right-hand side, we obtain the relation

$$
\begin{aligned}
& \frac{1}{\theta_{1} \theta_{2} \theta_{3}} J_{0}^{(d)}\left(\frac{\theta_{1}}{\theta_{2} \theta_{3}} m_{1}^{2}, \frac{\theta_{2}}{\theta_{1} \theta_{3}} m_{2}^{2}, \frac{\theta_{3}}{\theta_{1} \theta_{2}} m_{3}^{2}\right) \\
& =\frac{x_{1}}{\theta_{1} \theta_{4} \theta_{6}} J_{0}^{(d)}\left(\frac{\theta_{1}}{\theta_{4} \theta_{6}} m_{4}^{2}, \frac{\theta_{6}}{\theta_{1} \theta_{4}} m_{2}^{2}, \frac{\theta_{4}}{\theta_{1} \theta_{6}} m_{3}^{2}\right) \\
& \quad+\frac{x_{2}}{\theta_{2} \theta_{5} \theta_{6}} J_{0}^{(d)}\left(\frac{\theta_{6}}{\theta_{2} \theta_{5}} m_{1}^{2}, \frac{\theta_{2}}{\theta_{6} \theta_{5}} m_{4}^{2}, \frac{\theta_{5}}{\theta_{2} \theta_{6}} m_{3}^{2}\right) \\
& \quad+\frac{x_{3}}{\theta_{3} \theta_{4} \theta_{5}} J_{0}^{(d)}\left(\frac{\theta_{4}}{\theta_{3} \theta_{5}} m_{1}^{2}, \frac{\theta_{5}}{\theta_{3} \theta_{4}} m_{2}^{2}, \frac{\theta_{3}}{\theta_{5} \theta_{4}} m_{4}^{2}\right) .
\end{aligned}
$$

In terms of redefined masses $M_{1}, M_{2}, M_{3}$ related to original masses $m_{1}, m_{2}, m_{3}$ as

$$
m_{1}^{2}=\frac{\theta_{2} \theta_{3}}{\theta_{1}} M_{1}^{2}, \quad m_{2}^{2}=\frac{\theta_{1} \theta_{3}}{\theta_{2}} M_{2}^{2}, \quad m_{3}^{2}=\frac{\theta_{1} \theta_{2}}{\theta_{3}} M_{3}^{2}
$$

equation (5.27) reads

$$
\begin{aligned}
J_{0}^{(d)}\left(M_{1}^{2}, M_{2}^{2}, M_{3}^{2}\right)= & \frac{\theta_{2} \theta_{3}}{\widetilde{\theta}_{4} \widetilde{\theta}_{6}} \widetilde{x}_{1} J_{0}^{(d)}\left(\frac{\theta_{1}}{\widetilde{\theta}_{4} \widetilde{\theta}_{6}} m_{4}^{2}, \frac{\theta_{3} \widetilde{\theta}_{6}}{\theta_{2} \widetilde{\theta}_{4}} M_{2}^{2}, \frac{\theta_{2} \widetilde{\theta}_{4}}{\theta_{3} \widetilde{\theta}_{6}} M_{3}^{2}\right) \\
& +\frac{\theta_{1} \theta_{3}}{\widetilde{\theta}_{5} \widetilde{\theta}_{6}} \widetilde{x}_{2} J_{0}^{(d)}\left(\frac{\theta_{3} \widetilde{\theta}_{6}}{\theta_{1} \widetilde{\theta}_{5}} M_{1}^{2}, \frac{\theta_{2}}{\widetilde{\theta}_{5} \widetilde{\theta}_{6}} m_{4}^{2}, \frac{\theta_{1} \widetilde{\theta}_{5}}{\theta_{3} \widetilde{\theta}_{6}} M_{3}^{2}\right) \\
& +\frac{\theta_{1} \theta_{2}}{\widetilde{\theta}_{4} \widetilde{\theta}_{5}} \widetilde{x}_{3} J_{0}^{(d)}\left(\frac{\theta_{2} \widetilde{\theta}_{4}}{\theta_{1} \widetilde{\theta}_{5}} M_{1}^{2}, \frac{\theta_{1} \widetilde{\theta}_{5}}{\theta_{2} \widetilde{\theta}_{4}} M_{2}^{2}, \frac{\theta_{3}}{\widetilde{\theta}_{4} \widetilde{\theta}_{5}} m_{4}^{2}\right),
\end{aligned}
$$


where $\widetilde{x}_{i}, \widetilde{\theta}_{j}$ are defined from eqs. (5.11), (5.13) with redefined masses. After simplifications eq. (5.29) takes a simpler form:

$$
\begin{aligned}
J_{0}^{(d)} & \left(M_{1}^{2}, M_{2}^{2}, M_{3}^{2}\right) \\
= & J_{0}^{(d)}\left(\rho_{1}+\rho_{2}+M_{1}^{2}-M_{2}^{2}-M_{3}^{2}, \rho_{1}, \rho_{2}\right) \\
& +J_{0}^{(d)}\left(\rho_{3}, M_{3}^{2}-\rho_{2}, \rho_{3}-\rho_{2}-M_{1}^{2}+M_{2}^{2}\right) \\
& -J_{0}^{(d)}\left(\rho_{3}-M_{1}^{2}, \rho_{1}-M_{2}^{2}, \rho_{1}+\rho_{3}-M_{3}^{2}\right),
\end{aligned}
$$

where

$$
\begin{aligned}
& \rho_{1}=\frac{\left(M_{3}^{2}+M_{2}^{2}-M_{1}^{2}\right) \theta_{1} \theta_{2} \theta_{3}-2 b_{2} h_{2} \theta_{2} \theta_{3} m_{4}^{2}-\sqrt{\delta_{2}}}{2 \theta_{1} \theta_{2} \theta_{3} M_{2}^{2}-2 b_{2}^{2} \theta_{2}^{2} m_{4}^{2}} M_{2}^{2} \\
& \rho_{2}=\frac{\left(M_{3}^{2}+M_{2}^{2}-M_{1}^{2}\right) \theta_{1} \theta_{2} \theta_{3}-2 b_{2} h_{2} \theta_{2} \theta_{3} m_{4}^{2}+\sqrt{\delta_{2}}}{2 \theta_{1} \theta_{2} \theta_{3} M_{3}^{2}-2 h_{2}^{2} \theta_{3}^{2} m_{4}^{2}} M_{3}^{2} \\
& \rho_{3}=\frac{\left(M_{1}^{2}-M_{2}^{2}+M_{3}^{2}\right) \theta_{1} \theta_{2} \theta_{3}+2 a_{2} h_{2} \theta_{1} \theta_{3} m_{4}^{2}+\sqrt{\delta_{2}}}{2 \theta_{1} \theta_{2} \theta_{3} M_{1}^{2}-2 a_{2}^{2} \theta_{1}^{2} m_{4}^{2}} M_{1}^{2},
\end{aligned}
$$

with

$$
\begin{aligned}
\delta_{2} & =\theta_{1}^{2} \theta_{2}^{2} \theta_{3}^{2} \Delta_{2}+4 A \theta_{1} \theta_{2} \theta_{3} m_{4}^{2}, \\
\Delta_{2} & =M_{1}^{4}+M_{2}^{4}+M_{3}^{4}-2 M_{1}^{2} M_{2}^{2}-2 M_{1}^{2} M_{3}^{2}-2 M_{2}^{2} M_{3}^{2} .
\end{aligned}
$$

The coefficients $A, B$ and $C$ can be expressed in terms of $M_{i}, \theta_{j}$ :

$$
\begin{aligned}
& A=b_{2} h_{2} \theta_{2} \theta_{3} M_{1}^{2}-a_{2} h_{2} \theta_{1} \theta_{3} M_{2}^{2}+a_{2} b_{2} \theta_{1} \theta_{2} M_{3}^{2}, \\
& B=-\left(b_{1} h_{2}+b_{2} h_{1}\right) \theta_{2} \theta_{3} M_{1}^{2}+\left(a_{1} h_{2}+a_{2} h_{1}\right) \theta_{1} \theta_{3} M_{2}^{2}-\left(a_{1} b_{2}+a_{2} b_{1}\right) \theta_{1} \theta_{2} M_{3}^{2}, \\
& C=b_{1} h_{1} \theta_{2} \theta_{3} M_{1}^{2}-\theta_{1} \theta_{3} a_{1} h_{1} M_{2}^{2}+a_{1} b_{1} \theta_{1} \theta_{2} M_{3}^{2}-\theta_{1} \theta_{2} \theta_{3} m_{4}^{2} .
\end{aligned}
$$

One can easily observe that due to relations

$$
\begin{aligned}
& \rho_{1} \rho_{2}=M_{2}^{2} M_{3}^{3}, \\
& \rho_{1} \rho_{3}=M_{1}^{2} \rho_{1}+M_{2}^{2} \rho_{3}, \\
& \rho_{2} \rho_{3}=M_{3}^{2} \rho_{3}-M_{1}^{2} M_{3}^{2},
\end{aligned}
$$

parameters $\rho_{2}$ and $\rho_{3}$ can be expressed in terms of $\rho_{1}$ and therefore all the auxiliary parameters introduced in the derivation of the functional equation will be absorbed only in one parameter- $\rho_{1}$.

We would like to notice that eq. (5.30) is valid for integrals but not for their integrands. This is due to the fact that the factor in front of integral that comes from the scaling of $\alpha$ parameters in the parametric integral is not fully compensated by the scaling momenta given in eq. (5.26). 
At $m_{4}=0$ the dependence on all parameters $a_{i}, b_{j}, h_{k}$ in eqs. (5.29), (5.30) drops out and the integral $J_{0}^{(d)}\left(M_{1}^{2}, M_{2}^{2}, M_{3}^{2}\right)$ reduces to a combination of simpler integrals:

$$
\begin{aligned}
J_{0}^{(d)} & \left(M_{1}^{2}, M_{2}^{2}, M_{3}^{2}\right) \\
= & J_{0}^{(d)}\left(0, \frac{-M_{1}^{2}+M_{2}^{2}+M_{3}^{2}+\sqrt{\Delta_{2}}}{2}, \frac{-M_{1}^{2}+M_{2}^{2}+M_{3}^{2}-\sqrt{\Delta_{2}}}{2}\right) \\
& +J_{0}^{(d)}\left(\frac{M_{1}^{2}-M_{2}^{2}+M_{3}^{2}+\sqrt{\Delta_{2}}}{2}, 0, \frac{M_{1}^{2}-M_{2}^{2}+M_{3}^{2}-\sqrt{\Delta_{2}}}{2}\right) \\
& -J_{0}^{(d)}\left(\frac{-M_{1}^{2}-M_{2}^{2}+M_{3}^{2}+\sqrt{\Delta_{2}}}{2}, \frac{-M_{1}^{2}-M_{2}^{2}+M_{3}^{2}-\sqrt{\Delta_{2}}}{2}, 0\right) .
\end{aligned}
$$

The analytic expression for the integral $J_{0}^{(d)}$ with one mass equal to zero is known [14]. Under assumption that $\left|m_{3}^{2}-m_{2}^{2}\right| \leq\left|m_{3}^{2}\right|$ it reads

$$
J_{0}^{(d)}\left(0, m_{2}^{2}, m_{3}^{2}\right)=\frac{\pi^{2}(2-d)\left(m_{2}^{2} m_{3}^{2}\right)^{\frac{d-2}{2}}}{2(d-3) m_{3}^{2}\left(\Gamma\left(\frac{d}{2}\right) \sin \frac{\pi d}{2}\right)^{2}} \quad{ }_{2} F_{1}\left[\begin{array}{c}
1,2-\frac{d}{2} ; 1-\frac{m_{2}^{2}}{m_{3}^{2}} \\
4-d ;
\end{array}\right] .
$$

From functional equation (5.35) as a by-product one can get a new hypergeometric representation for the one-loop massless vertex type integral. In ref. [15] an interesting relation between the dimensionally regularized one-loop vertex type integral $I_{3}^{(d)}\left(m_{1}^{2}, m_{2}^{2}, m_{3}^{2}\right)$ and the two-dimensional integral $J_{0}^{(6-d)}\left(m_{1}^{2}, m_{2}^{2}, m_{3}^{2}\right)$ was discovered

$$
I_{3}^{(d)}\left(s_{23}, s_{13}, s_{12}\right)=\frac{\Gamma\left(3-\frac{d}{2}\right)}{\Gamma(d-3)}\left(-s_{23} s_{13} s_{12}\right)^{\frac{d-4}{2}} J_{0}^{(6-d)}\left(s_{23}, s_{13}, s_{12}\right) .
$$

The functional equation (5.35) with $J_{0}^{(d)}\left(0, m_{2}^{2}, m_{3}^{2}\right)$ defined in eq. (5.36) provides us with a new hypergeometric representation for the integral $I_{3}^{(d)}$ with massless propagators. Formula for the one-loop massless vertex integral in terms of other Gauss' hypergeometric functions is given in ref. [16].

\section{Conclusions}

Finally, we summarize what we have accomplished in this paper.

First of all, we formulated new methods for deriving functional equations for Feynman integrals. These methods are quite simple and do not use any kind of integration by parts techniques.

Second, it has been shown that integrals with many kinematic arguments can be reduced to a combination of simpler integrals with fewer arguments. In our future publications we are going to demonstrate that in some cases applying functional equations one can reduce, the so-called, master integrals to a combination of simpler integrals from, what we would like to call, a 'universal' basis of integrals.

The method based on algebraic relations for 'deformed propagators' can be used not only for the vacuum type integrals, but also for the integrals depending on external momenta. In this paper we consider rather particular cases of the functional equations. The 
systematic investigation and classification of the proposed functional equations requires application of the methods of algebraic geometry and group theory.

At the moment, it is not quite clear whether functional equations derivable from recurrence relations can be reproduced by the methods of algebraic relations between products of propagators described in section 3 and section 5 .

A detailed consideration of our functional equations and their application to the oneloop integrals with four, five and six external legs as well as to some two- and three-loop Feynman integrals will be presented in future publications.

Finally, we would like to make a short comparison of our functional equations and the relationship presented in ref. [4]. The relationship given in ref. [4] corresponds to a particular choice of arbitrary parameters appearing in our functional equations. It has no free parameters. In general, our functional equations depend on several arbitrary parameters. This arbitrariness allows one to analytically continue the integrals to the required regions of kinematical variables.

In our approach, by choosing the arbitrary parameters, one can get also relationships between the integrals depending only on the arguments that are free of square roots of kinematical determinants. This was explicitely demonstrated in ref. [2].

\section{Acknowledgments}

This work was supported by the German Science Foundation (DFG) within the Collaborative Research Center 676 Particle, Strings and the Early Universe: the Structure of Matter and Space-Time. I am thankful to O.L. Veretin for providing results for integrals contributing to ortho-positronium lifetime described in ref. [13].

Open Access. This article is distributed under the terms of the Creative Commons Attribution License (CC-BY 4.0), which permits any use, distribution and reproduction in any medium, provided the original author(s) and source are credited.

\section{References}

[1] O.V. Tarasov, New relationships between Feynman integrals, Phys. Lett. B 670 (2008) 67 [arXiv:0809.3028] [INSPIRE].

[2] O.V. Tarasov, Functional equations for Feynman integrals, Phys. Part. Nucl. Lett. 8 (2011) 419 [InSPIRE].

[3] B.A. Kniehl and O.V. Tarasov, Functional equations for one-loop master integrals for heavy-quark production and Bhabha scattering, Nucl. Phys. B 820 (2009) 178 [arXiv:0904.3729] [INSPIRE].

[4] A.I. Davydychev and R. Delbourgo, A Geometrical angle on Feynman integrals, J. Math. Phys. 39 (1998) 4299 [hep-th/9709216] [InSPIRE].

[5] A.I. Davydychev, Geometrical splitting and reduction of Feynman diagrams, J. Phys. Conf. Ser. 762 (2016) 012068 [arXiv: 1605.04828] [INSPIRE].

[6] O.V. Tarasov, Connection between Feynman integrals having different values of the space-time dimension, Phys. Rev. D 54 (1996) 6479 [hep-th/9606018] [INSPIRE]. 
[7] J. Fleischer, F. Jegerlehner and O.V. Tarasov, Algebraic reduction of one loop Feynman graph amplitudes, Nucl. Phys. B 566 (2000) 423 [hep-ph/9907327] [INSPIRE].

[8] Z. Bern, L.J. Dixon and D.A. Kosower, Dimensionally regulated one loop integrals, Phys. Lett. B 302 (1993) 299 [Erratum ibid. B 318 (1993) 649] [hep-ph/9212308] [InSPIRE].

[9] Z. Bern, L.J. Dixon and D.A. Kosower, Dimensionally regulated pentagon integrals, Nucl. Phys. B 412 (1994) 751 [hep-ph/9306240] [INSPIRE].

[10] G. 't Hooft and M.J.G. Veltman, Scalar One Loop Integrals, Nucl. Phys. B 153 (1979) 365 [INSPIRE].

[11] O.V. Tarasov, Generalized recurrence relations for two loop propagator integrals with arbitrary masses, Nucl. Phys. B 502 (1997) 455 [hep-ph/9703319] [INSPIRE].

[12] D.J. Broadhurst, J. Fleischer and O.V. Tarasov, Two loop two point functions with masses: Asymptotic expansions and Taylor series, in any dimension, Z. Phys. C 60 (1993) 287 [hep-ph/9304303] [INSPIRE].

[13] B.A. Kniehl, A.V. Kotikov and O.L. Veretin, Irrational constants in positronium decays, arXiv:0811.0306 [INSPIRE].

[14] A.I. Davydychev and J.B. Tausk, Two loop selfenergy diagrams with different masses and the momentum expansion, Nucl. Phys. B 397 (1993) 123 [INSPIRE].

[15] A.I. Davydychev and J.B. Tausk, A magic connection between massive and massless diagrams, Phys. Rev. D 53 (1996) 7381 [hep-ph/9504431] [INSPIRE].

[16] A.I. Davydychev, Explicit results for all orders of the $\epsilon$-expansion of certain massive and massless diagrams, Phys. Rev. D 61 (2000) 087701 [hep-ph/9910224] [INSPIRE]. 